\title{
Research on micro-Doppler feature of spatial target
}

\author{
Zhenyuan Ji (D), Encheng Hu, Yun Zhang and Hongyan Jin
}

\begin{abstract}
The micro-Doppler ( $m-D)$ effect which is also called micro-motion effect is a kind of physical phenomenon, which is often produced in ISAR imaging of space targets. When the space target has a micro-Doppler effect, it will produce frequency modulation for the radar echo and cause serious interference to ISAR imaging of the target. In this paper, the micro-spin model is modeled, and the echo of micro-spin model scattering point is processed respectively by short-time Fourier transform (STFT), Wigner-Vile Distribution (WVD), and pseudo-Wigner-Vile Distribution (PWV), and the micro-Doppler curve is derived. On this basis, for the space target with spin components, this paper introduces an ISAR imaging algorithm using the micro-Doppler removal based on STFT and proposes an improved algorithm based on rigid body parameter estimation, which can obtain better imaging results.
\end{abstract}

Keywords: Micro-Doppler, Time-frequency analysis, ISAR imaging algorithm, Rigid body parameter estimation

\section{Introduction}

The concept of micro-Doppler (m-D) is firstly proposed by V.C. Chen of the US Naval Research Laboratory [1], which collectively refers to tiny movements such as vibration, rotation, and accelerated movement other than some parts of the target or target. And the modulation of these tiny movements on the radar echo spectrum is called the micro-Doppler effect.

The physical essence of micro-Doppler is the Doppler effect of the relative distance between the equivalent scattering center of the target and the phase center of the radar antenna as the micro-motion changes. Under the condition of small relative measurement bandwidth and observation accumulation angle, the local scattering source of the target can be approximated by the ideal point scattering center. The scattering center has a constant position and phase, and the scattering intensity varies little with frequency. This approximation is the basis of the current micro-Doppler mechanism research on target characteristics but also the basis of the establishment of micro-Doppler mathematical model [1]. From the literature point of view, the research contents of radar micro-moving target characteristics can be summarized as micro-target radar echo modeling, microDoppler signal separation, micro-Doppler feature extraction, micro-motion target imaging, and micro-Doppler characteristics of the target classification and identification [2].

\section{Construction of point scattering model for micro-spin target}

Spin is a basic form of micro-movement but also the common form of space target micro-movement. We can create three coordinate systems: radar coordinate system $(U, V, W)$, reference coordinate system $(X, Y$, $Z)$, and local coordinate system $(x, y, z)$. The geometric relationship between the rotating target of the radar target is shown in Fig. 1.

A rigid body rotates at the angular velocity $\vec{\omega}=\left(\omega_{x}, \omega_{y}, \omega_{z}\right)^{T}$ along the coordinate axes $x, y$, and $z$ in the local coordinate system $(x, y, z)$, the initial position of a scattering point $\mathrm{P}$ on the rigid body is $\overrightarrow{r 0}=\left(\omega_{x}, \omega_{y}, \omega_{z}\right)^{T}$, its coordinates in the reference coordinate system $(X, Y, Z)$ is $\mathfrak{R}_{\text {Init }} \cdot \overrightarrow{r_{0}}$, and $\mathfrak{R}_{\text {Init }}$ is the initial rotation matrix.

\footnotetext{
* Correspondence: jizhenyuan@hit.edu.cn

Harbin Institute of Technology, Xidazhijie, 92, Harbin 150006, China
} 


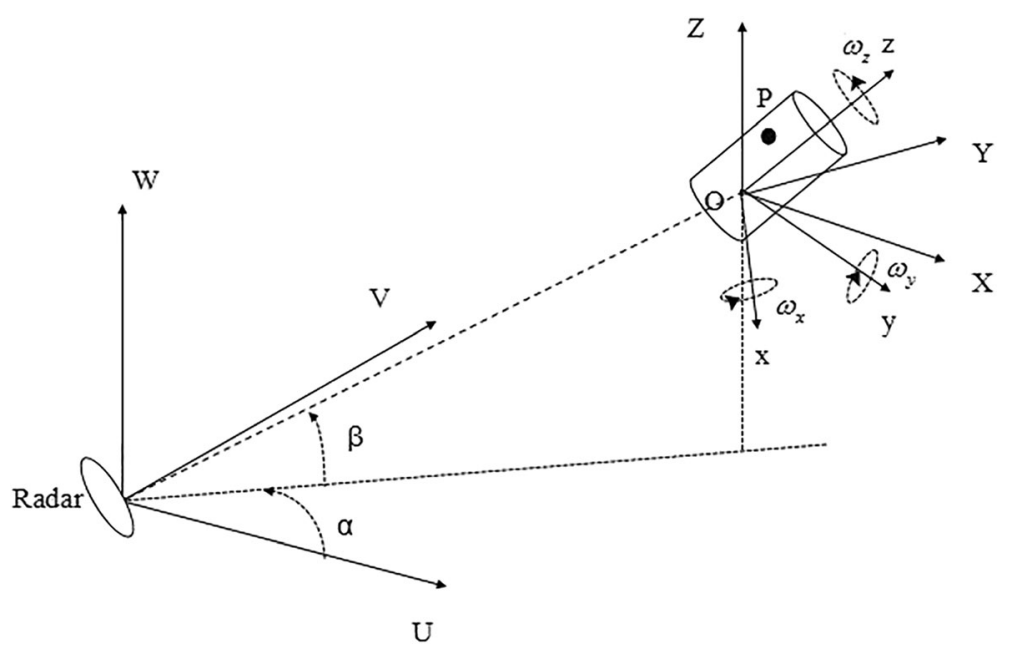

Fig. 1 The geometric representation of micro-spin target

$$
\mathfrak{R}_{\text {Init }}=\left[\begin{array}{ccc}
\cos \phi & -\sin \phi & 0 \\
\sin \phi & \cos \phi & 0 \\
0 & 0 & 1
\end{array}\right]\left[\begin{array}{ccc}
1 & 0 & 0 \\
0 & \cos \theta & -\sin \theta \\
0 & \sin \theta & \cos \theta
\end{array}\right]\left[\begin{array}{ccc}
\cos \psi & -\sin \psi & 0 \\
\sin \psi & \cos \psi & 0 \\
0 & 0 & 1
\end{array}\right]
$$

The rotation unit vector in the reference coordinate system is:

$$
\vec{\omega}=\left(\omega_{x}, \omega_{y}, \omega_{z}\right)^{T}=\frac{\boldsymbol{R}_{\text {Init }} \cdot \vec{\omega}}{\|\vec{\omega}\|}
$$

According to the Roche equation, at time $t_{m}$, the rotation vector in the reference coordinate system is:

$$
\boldsymbol{R}_{t}=I+\hat{\omega}^{\prime} \sin \left(\Omega t_{m}\right)+\hat{\omega}^{\prime 2}\left(1-\cos \Omega t_{m}\right)
$$

where $\omega$ is an oblique symmetric array.

$$
\omega^{\prime}=\left[\begin{array}{ccc}
0 & -\omega_{z}^{\prime} & \omega_{y}^{\prime} \\
\omega_{z}^{\prime} & 0 & -\omega_{x}^{\prime} \\
-\omega_{y}^{\prime} & \omega_{x}^{\prime} & 0
\end{array}\right]
$$

So, the vector of point $\mathrm{P}$ in the reference coordinate system at time $t_{m}$ is:

$$
\vec{r}=\mathfrak{R}_{t} \cdot \mathfrak{R}_{\text {Init }} \cdot \overrightarrow{r_{0}}
$$

The coordinate vector of point $\mathrm{P}$ in the radar coordinate system is:

$$
\vec{R}_{t}=\overrightarrow{\mathrm{R}}+\vec{r}=\vec{R}+\boldsymbol{R}_{t} \cdot \boldsymbol{R}_{\text {Init }} \cdot \overrightarrow{r_{0}}
$$

where $\vec{R}$ is the position of the origin of the reference coordinate system in the radar coordinate system.

From the ISAR imaging principle, the expression of the echo signal of the single-base broadband ISAR imaging radar in the fast time domain is:

$$
\mathrm{S}_{d}\left(\mathrm{f}_{k}, t_{m}\right)=\sigma \mathrm{T}_{p} \sin c\left[T_{p}\left(\mathrm{f}_{k}+\frac{2 K_{r} \Delta R\left(t_{m}\right)}{c}\right)\right] \exp \left\{-j \frac{4 \pi}{\lambda} \Delta R\left(t_{m}\right)\right\}
$$

where $t_{m}$ is the slow time, $T_{p}$ is the pulse signal repetition period, and $K_{r}$ is the frequency modulation slope. $\Delta R\left(t_{m}\right)=R\left(t_{m}\right)-R_{r e f}\left(t_{m}\right), R\left(t_{m}\right)$ is the distance from the target to a radar at time $t_{m}$, and $R_{r e f}\left(t_{m}\right)$ is the distance from the target center to the radar at time $t_{m}$.

Assume that the radar line of sight LOS direction unit vector is $\vec{n}$, its micro-Doppler can be approximated as:

$$
\begin{aligned}
f_{m D}\left(t_{m}\right) & =\frac{2}{\lambda} \frac{d \Delta R\left(t_{m}\right)}{d t_{m}} \\
& =\frac{2 \Omega}{\lambda}\left[\hat{\omega}\left(\hat{\omega} \sin \Omega t_{m}+I \cos \Omega t_{m}\right) \Re_{\text {Init }} \cdot \overrightarrow{r_{0}}\right]^{T} \cdot \vec{n}
\end{aligned}
$$

which can be written as:

$$
f_{m D}\left(t_{m}\right)=A_{1} \cos \Omega t_{m}+A_{2} \sin \Omega t_{m}
$$

where $A_{1}=\frac{2 \Omega}{\lambda}\left[\hat{\omega^{\prime}} \Re_{\text {Init }} \cdot \vec{r}_{0}\right]^{T} \cdot \vec{n}, A_{2}=\frac{2 \Omega}{\lambda}\left[\hat{\omega}^{\prime 2} \Re_{\text {Init }} \cdot \vec{r}_{0}\right]^{T} \cdot \vec{n}$.

It can be seen that the rotating micro-Doppler is related to the Euler angle, the rotational angular velocity and the initial distance, so the target ISAR radar echo can be written as:

$$
\mathrm{S}_{d}\left(\mathrm{f}_{k 0}, t_{m}\right)=\sigma \mathrm{T}_{p} \exp \left\{-j \phi_{d}\left(\mathrm{t}_{m}\right)\right\}
$$

$\phi_{d}\left(t_{m}\right)$ is the Doppler phase shift produced by the target micro-motion. Equation (10) is the echo model of the micro spin target.

\section{Time-frequency analysis of micro-Doppler}

Time-frequency analysis is also called time-frequency distribution. Its basic task is to describe the energy distribution density of the signal in time and frequency and 


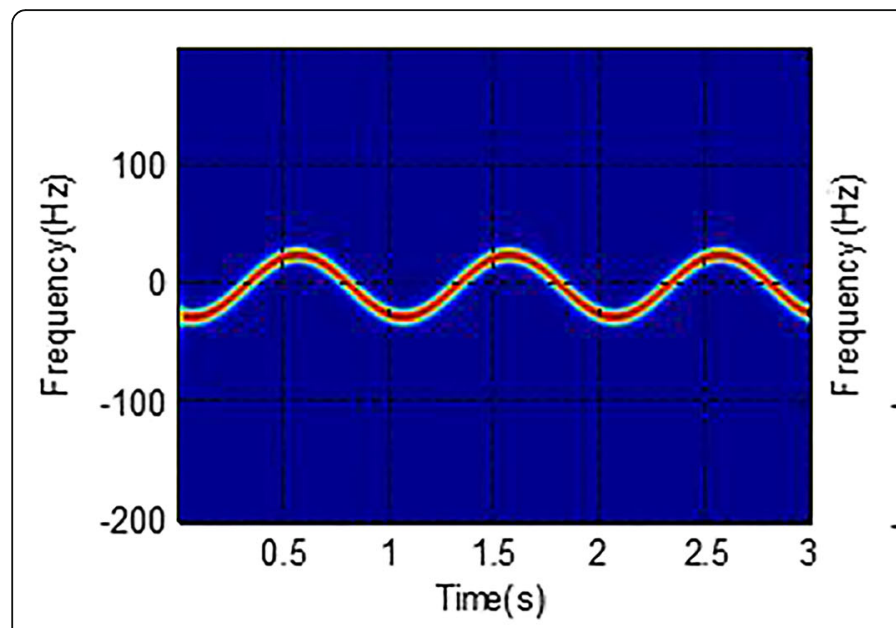

(a) STFT analysis curve

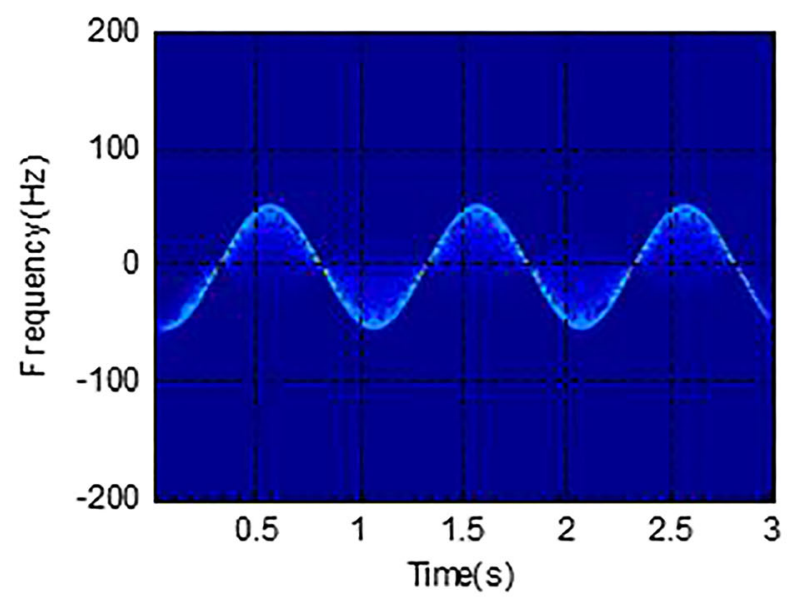

(c) PWV analysis curve

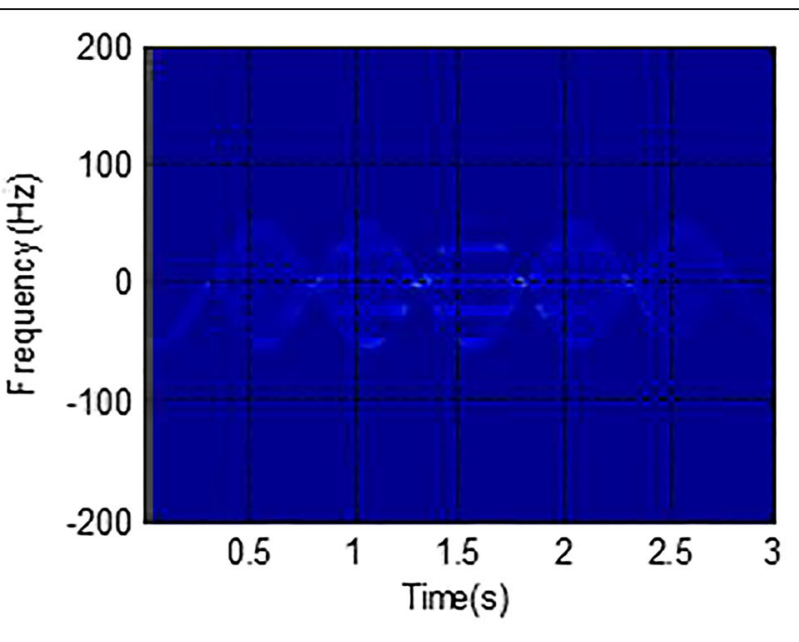

(b) WVD analysis curve

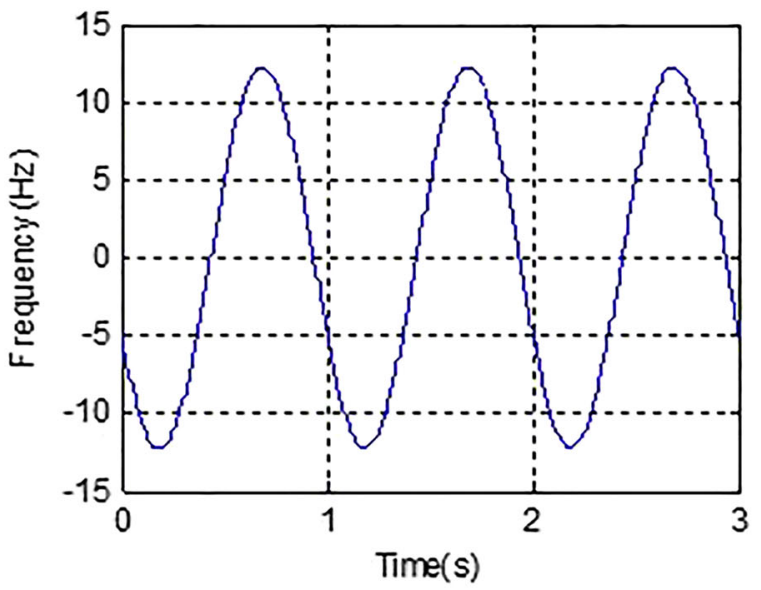

(d) Theoretical micro-Doppler curve

Fig. 2 Spin-fretting model time-frequency analysis m-D graph. a STFT analysis curve. b WVD analysis curve. c PW analysis curve. d Theoretical micro-Doppler curve

describe the law that the frequency of the signal changes with time $[3,4]$. It uses time-frequency to characterize the signal. We can master the time domain information but also master the frequency domain information; we can also know a frequency component at which moment and how it changes, which can fully observe the signal characteristics. Commonly used time-frequency analysis methods include short-time Fourier transform, Wignervile distribution, and pseudo-Wigner-vile distribution.

The single-component signal of the spin-moving target point is simulated, and then, the signal is processed by time-frequency analysis method. The simulation parameters are radar transmission signal-carrier frequency $f=10 \mathrm{GHz}$, bandwidth $B=500 \mathrm{MHz}$, and pulse repetition frequency $f_{P}=500 \mathrm{~Hz}$. The coordinates of the target in the radar coordinate system are $(X=0, Y=2.53, Z=4.38) \mathrm{km}$.
It is assumed that the azimuth and pitch angles of the target center of mass relative to the radar coordinate system are $\alpha$ $=\pi / 2$ and $\beta=\pi / 3$, assume that the target has a scattering point $\mathrm{P}$ with coordinates $(x=1.0, y=0.4, z=0.6) \mathrm{m}$, the initial Euler angle of the target local coordinate system is $(\phi=30, \theta=20, \psi=20)$, The angular velocity of point $\mathrm{P}$ is $\vec{\omega}=(\pi, \sqrt{2} \pi, \pi)^{T} \mathrm{rad} / \mathrm{s}$ The theoretical micro-Doppler curve and time-frequency analysis are shown in Fig. 2.

Simulation results show that the STFT has the best analysis effect in the three time-frequency analysis methods. Through the micro-Doppler curve, the micro-Doppler law can be seen intuitively. The effect of the WVD in the three time-frequency analysis methods is not ideal, and there is other timefrequency curve interference in the time-frequency diagram, which is generated by the cross-term of the 


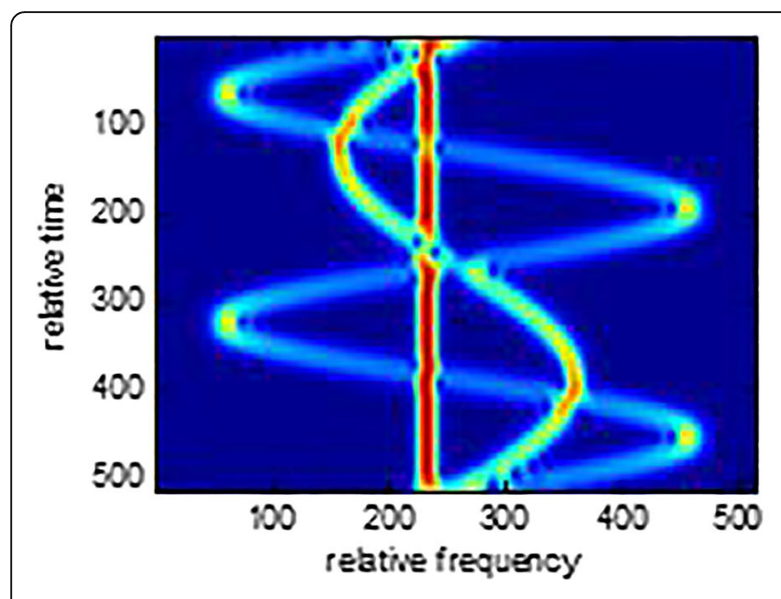

(a) STFT of the signal

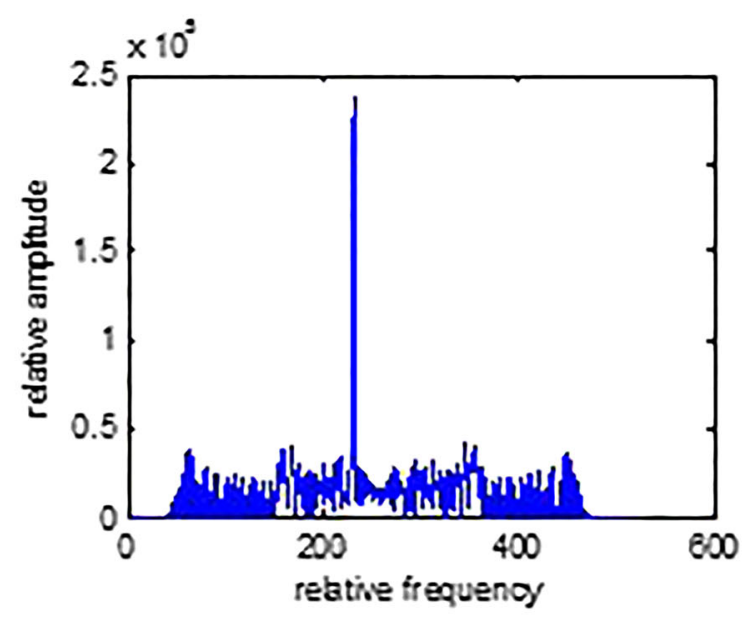

(c) Reconstructed FFT

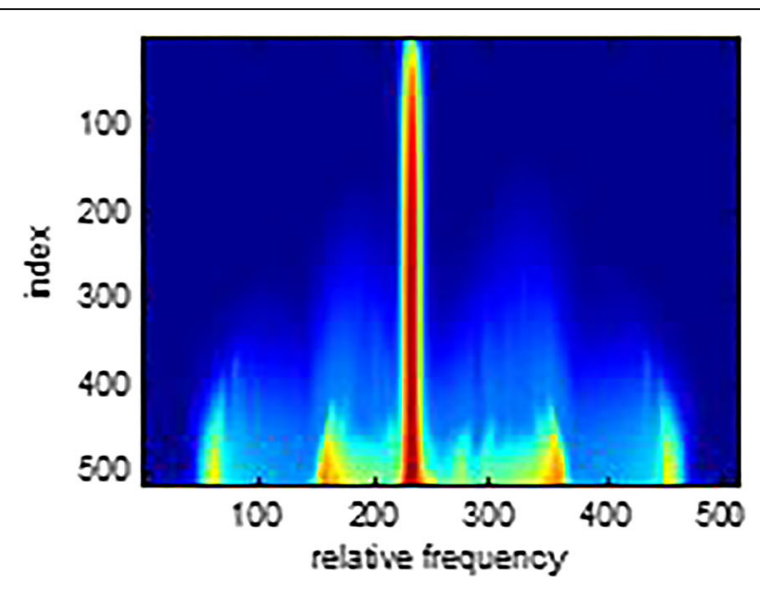

(b) The rearrangement of STFT

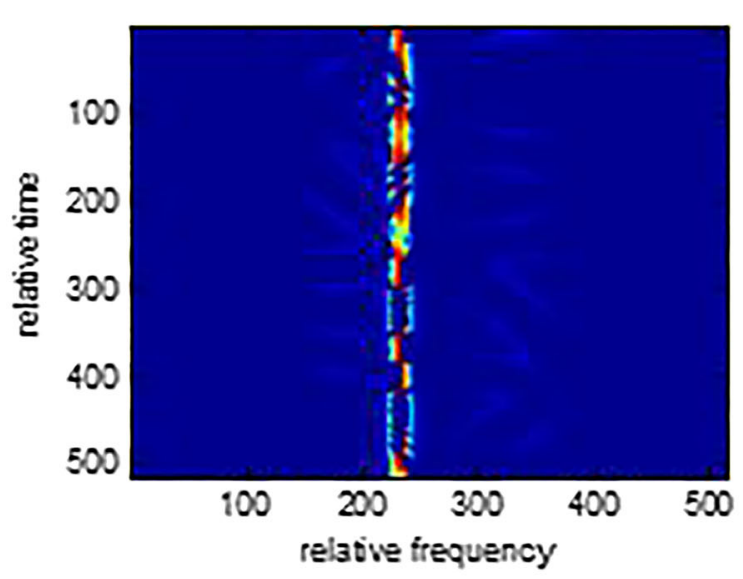

(d) STFT of the signal after $m-D$ removal

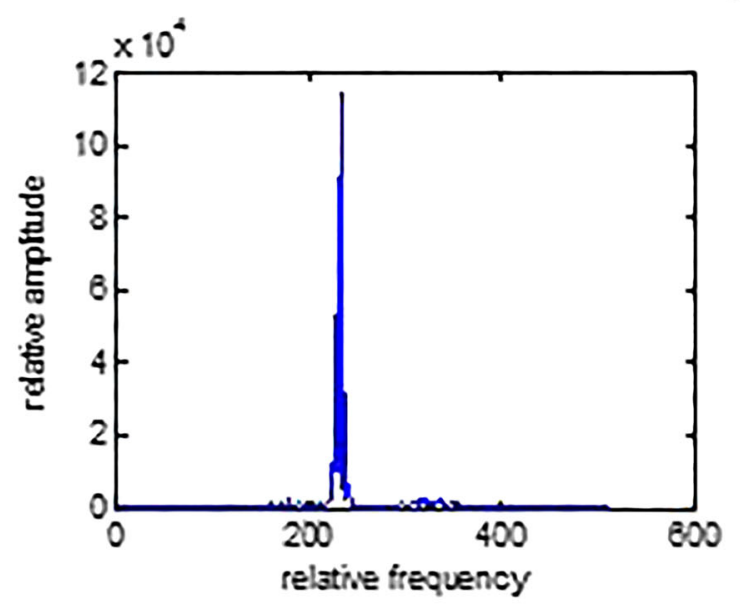

\section{(e) FFT of the signal after m-D removal}

Fig. 3 Signal model simulation results. a STFT of the signal. $\mathbf{b}$ The rearrangement of STFT. c Reconstructed FFT. $\mathbf{d}$ STFT of the signal after m-D removal. e FFT of the signal after m-D removal 
signal in the transformation. The time-frequency diagram appears fuzzy now. The effect of PWV in the three time-frequency analysis methods is centered. Due to the smoothing of the window, the fuzzy phenomenon of the time-frequency diagram is greatly reduced, and the interference of other time-frequency curves can be eliminated. The change of microDoppler is better observed [5-7].

\section{ISAR imaging algorithm for space targets with rotating parts}

ISAR imaging of space targets with moving parts usually produces micro-Doppler phenomena, which reduce the image quality of ISAR and interfere with the resolution of the target $[8,9]$. In this paper, we introduce an ISAR imaging algorithm which can effectively remove the micro-Doppler effect. The algorithm can effectively eliminate the effect of jog effect by shorttime Fourier transform (STFT) and get good results in ISAR imaging.

\subsection{ISAR imaging algorithm of $m-D$ removal based on} STFT

The discretization form of short-time Fourier transform (STFT) can be expressed as:

$$
\operatorname{STFT}(m, k)=\sum_{i=1}^{M-1} s(i) w(i-m) e^{-j 2 \pi i k / m}
$$

Consider a set of $M$ elements of the STFT, for a certain frequency $k$ :

$$
S_{k}(m)=\{\operatorname{STFT}(m, k), m=0,1, \ldots, M-1\}
$$

By the sorting procedure, we can get a new ordered set of elements:

$\Psi_{k}(m) \in S_{k}(m)$, such as $\left|\Psi_{k}(0)\right| \leq\left|\Psi_{k}(1)\right| \leq\left|\Psi_{k}(2)\right| \ldots \leq \mid$ $\Psi_{k}(M-1) \mid$. Summing all the STFT samples, we obtain:

$$
S_{L}(k)=\sum_{M=0}^{M_{Q}-1} \Psi_{k}(m)
$$

where $M_{Q}=\operatorname{int}[M(1-Q / 100)]$ and $\mathrm{Q}$ is the percent of removed values. Equation (13) is the reconstructed FFT of the signal.

After the above-mentioned processing, the singlefrequency component and the sinusoidal frequency modulation component of the signal can be effectively separated; we can use this principle to simulate the signal analysis.

From the previous analysis, it can be seen that the echo signal of the target with rotating parts not only contains the single-frequency component but also the

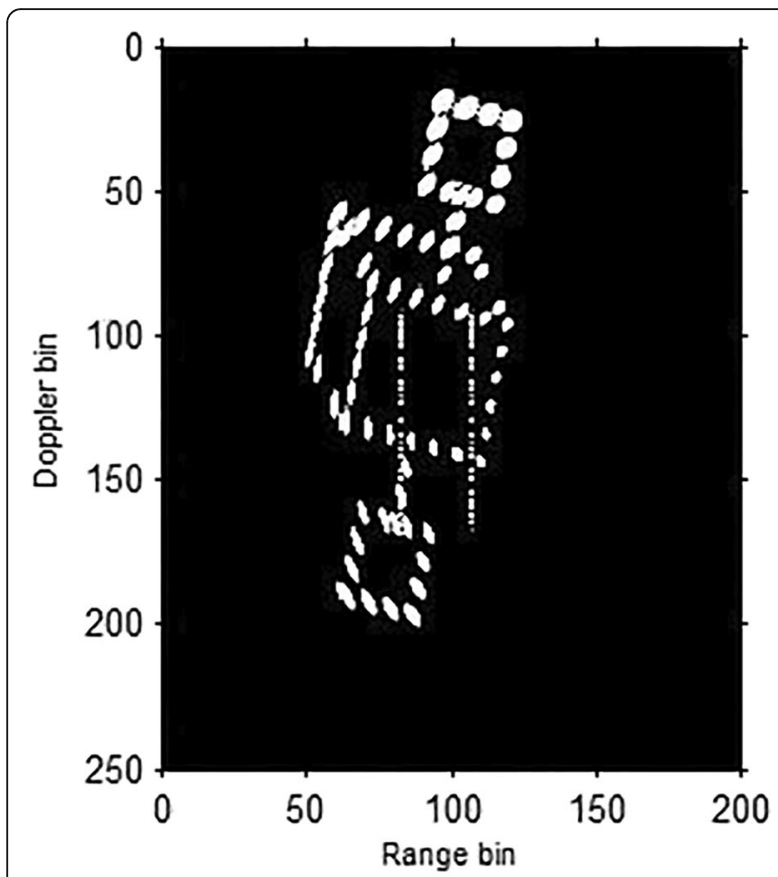

(a) Result of R-D algorithm

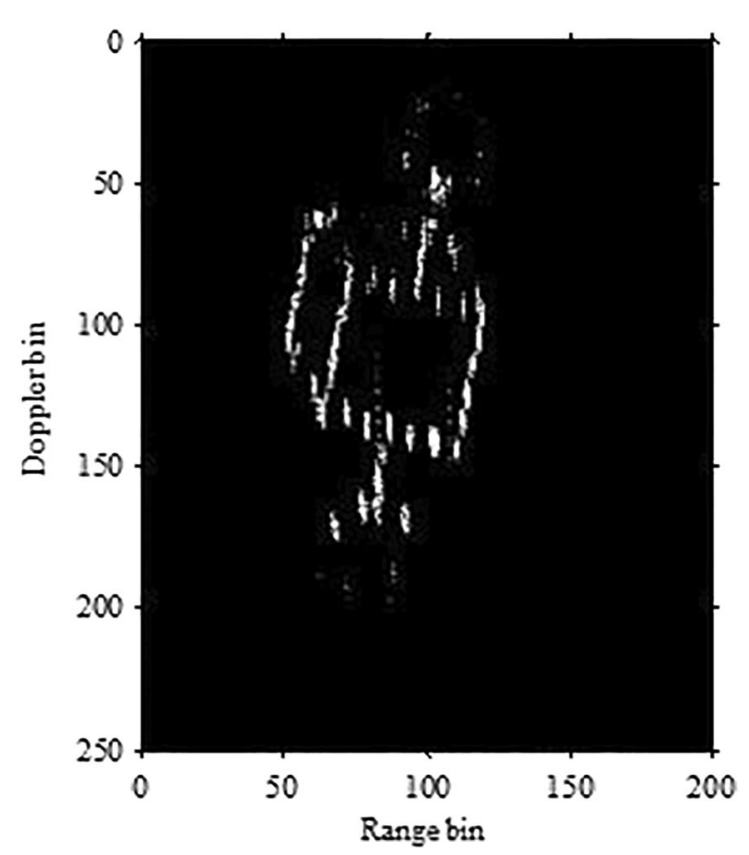

(b) Result of $\mathrm{m}-\mathrm{D}$ removal algorithm

Fig. 4 Simulation results of imaging algorithm. a Result of R-D algorithm. b Result of m-D removal algorithm 
sine frequency modulation component, so let the signal model be:

$$
\begin{aligned}
s(m) & =\sigma_{B} \sum_{i=1}^{K} e^{j y_{B i} m} \\
& +\sigma_{R} \sum_{i=1}^{P} e^{j\left[y_{R O i} m+A_{R i} \sin \left(\omega_{R i} m+\phi_{i}\right)\right]}
\end{aligned}
$$

Let $K=1, P=2, \sigma_{B}=20, \sigma_{R}=15, y_{B 1}=0.1 \pi, A_{R i}=100$, $\omega_{R 1}=\pi / 256, \omega_{R 2}=\pi / 128, y_{R O i}=0, \phi_{i}=0$. The signal contains a single-frequency signal and three sinusoidal FM signals. The simulation results are shown in Fig. 3.

By comparing the simulation results in Fig. 3c, e, we can see that this method has a good effect on the separation of the single-frequency signal and the sinusoidal frequency modulation signal. Here, we apply this method to the space target with the rotating part. In ISAR imaging, the algorithm steps are as follows:
Step 1 For the echo signal in each range bin, calculate STFT values of mixed signals. By STFT, the mixed signals are demonstrated in the T-F domain.

Step 2 At each frequency bin of the TF domain, sort the absolute values of STFT results.

Step 3 Remove the sorting STFT results according to the constant threshold such as $50 \%$ of the highest value part of the TF domain or an adaptive threshold in equation 14.

Step 4 Obtain rigid body by summing the remaining STFT results.

Using this algorithm to simulate, the simulation target is a low-orbit satellite, assuming that the target has completed the speed compensation and motion compensation. Assuming that there are two points on the satellite in a micro-spin state, the rotation frequency is 4 and $6 \mathrm{~Hz}$, respectively, and the radius of rotation is 0.3 and $0.5 \mathrm{~m}$ respectively. The simulation parameters are as follows: bandwidth is $500 \mathrm{MHz}$, carrier frequency is $10 \mathrm{GHz}$, pulse repetition frequency is $500 \mathrm{~Hz}$, horizontal longitudinal sampling points are 256 points. The simulation results are shown in Fig. 4.
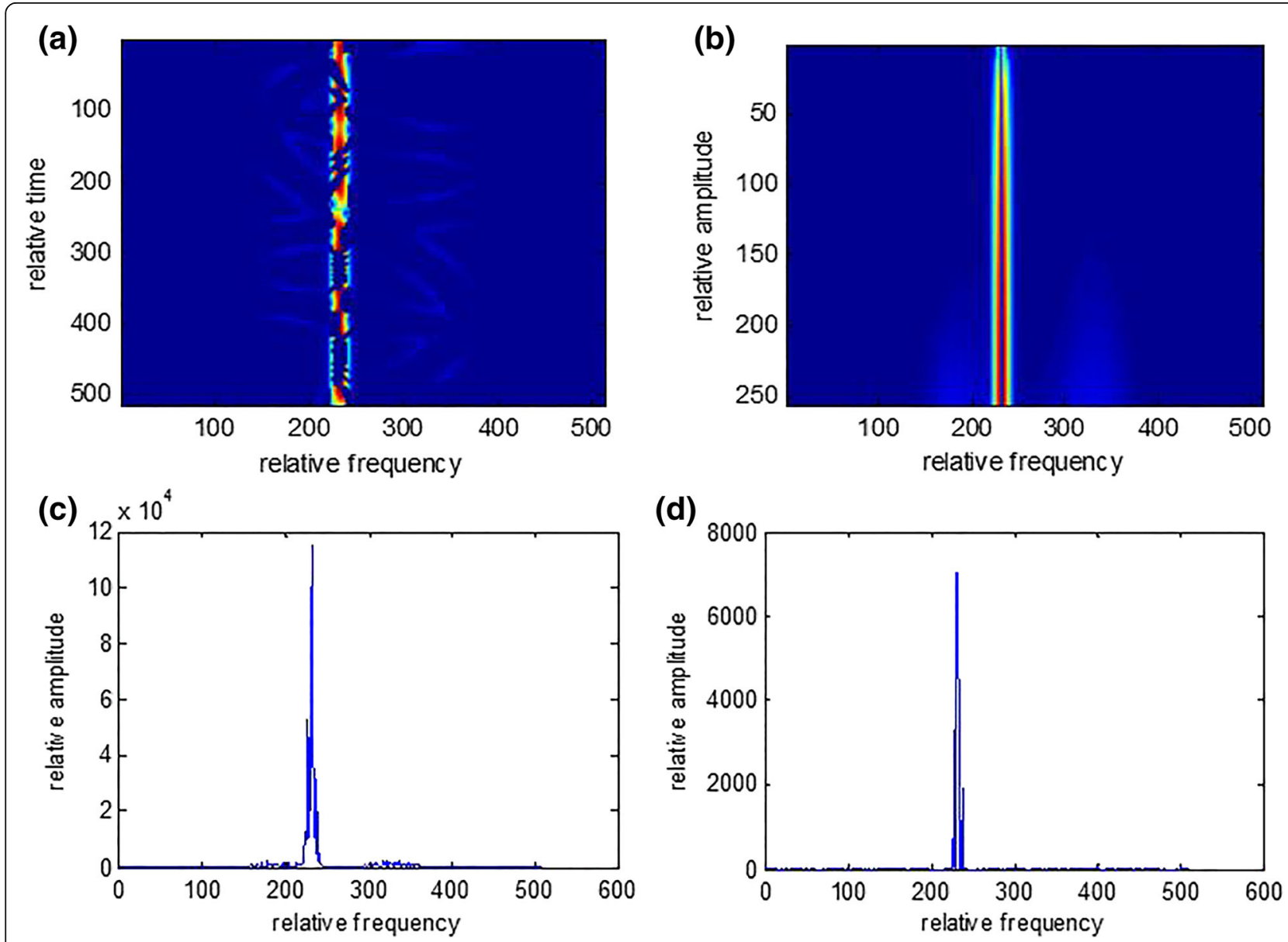

Fig. 5 Signal model simulation results of the proposed algorithm. a STFT of the signal after m-D removal in Section 4.1. $\mathbf{b}$ STFT of the signal after m-D removal with the proposed algorithm. c Reconstructed FFT in Section 4.1. d Reconstructed FFT with the proposed algorithm 
From Fig. 4a, it can be found that the space target with the spin component has two obvious interference lines in the middle of the image when the RD imaging algorithm is used. This is the spectrum of the sine FM signal generated by the spin component. Figure $4 \mathrm{~b}$ shows that the effect of the fretting effect can be effectively eliminated by using the micro-Doppler removal ISAR imaging algorithm proposed in this section, but its image resolution is reduced and some of the rigid body information of satellite is missing.

\subsection{An improved algorithm based on rigid body parameter estimation}

From the signal model simulation results shown in Fig. 3, we can see that the main lobe of the recovered singlefrequency signal is widened and has obvious side lobes, this is because the most important step of the method described in Section 4.1 is to remove the larger amplitude elements in the time-frequency plane. However, this operation also eliminates some of the elements of the single-frequency signal in the time-frequency plane, which manifests the widening of the main lobe width of the recovered and the presence of the side lobes.

After the removal of the micro-Doppler interference, the cumulative sum of the remaining elements at the rigid scattering point $k$ is expressed as:

$$
\begin{aligned}
S_{L}\left(k_{0}\right) & =\sigma \sum_{m=0}^{M_{Q}-1} W(0) e^{j 2 \pi m \times 0 / M} \\
& =\sigma \times M_{Q} \times W(0)
\end{aligned}
$$

$\sigma$ is the amplitude of the single-frequency signal corresponding to the single-frequency signal, $W(0)$ is the value of the Fourier transform at the zero point of the time-frequency analysis, and $M_{Q}$ is the number of the remaining elements. Since these three parameters are fixed values, we can estimate the corresponding singlefrequency signal amplitude value $S_{L}(k)$ by finding the value at the scattering point of the rigid body, and the frequency value of the single-frequency signal can be estimated by $k_{0}$ so that we can estimate the singlefrequency signal corresponding to the rigid body scattering point.

The simulated signal in Section 4.1 is simulated and analyzed by this principle. The simulation results are shown in Fig. 5.

Figure 5a shows that the direct removal of the microDoppler part also causes the loss of the single-frequency signal component. Figure $5 \mathrm{c}$ shows that the singlefrequency signal recovered directly from Fig. 5a will cause the main lobe to widen due to the absence of data; Fig. $5 \mathrm{~b}, \mathrm{~d}$ shows that the improved algorithm proposed in this section can solve the above problem effectively.
Here, we apply this improved algorithm to the spatial target ISAR imaging with spin components. The algorithm steps are as follows:

Step 1 For the echo signal in each range bin, calculate STFT values of mixed signals. By STFT, the mixed signals are demonstrated in the T-F domain.

Step 2 At each frequency bin of the TF domain, sort the absolute values of STFT results.

Step 3 Remove the sorting STFT results according to the constant threshold such as $50 \%$ of the highest value part of the TF domain.

Step 4 Obtain rigid body by summing the remaining STFT results.

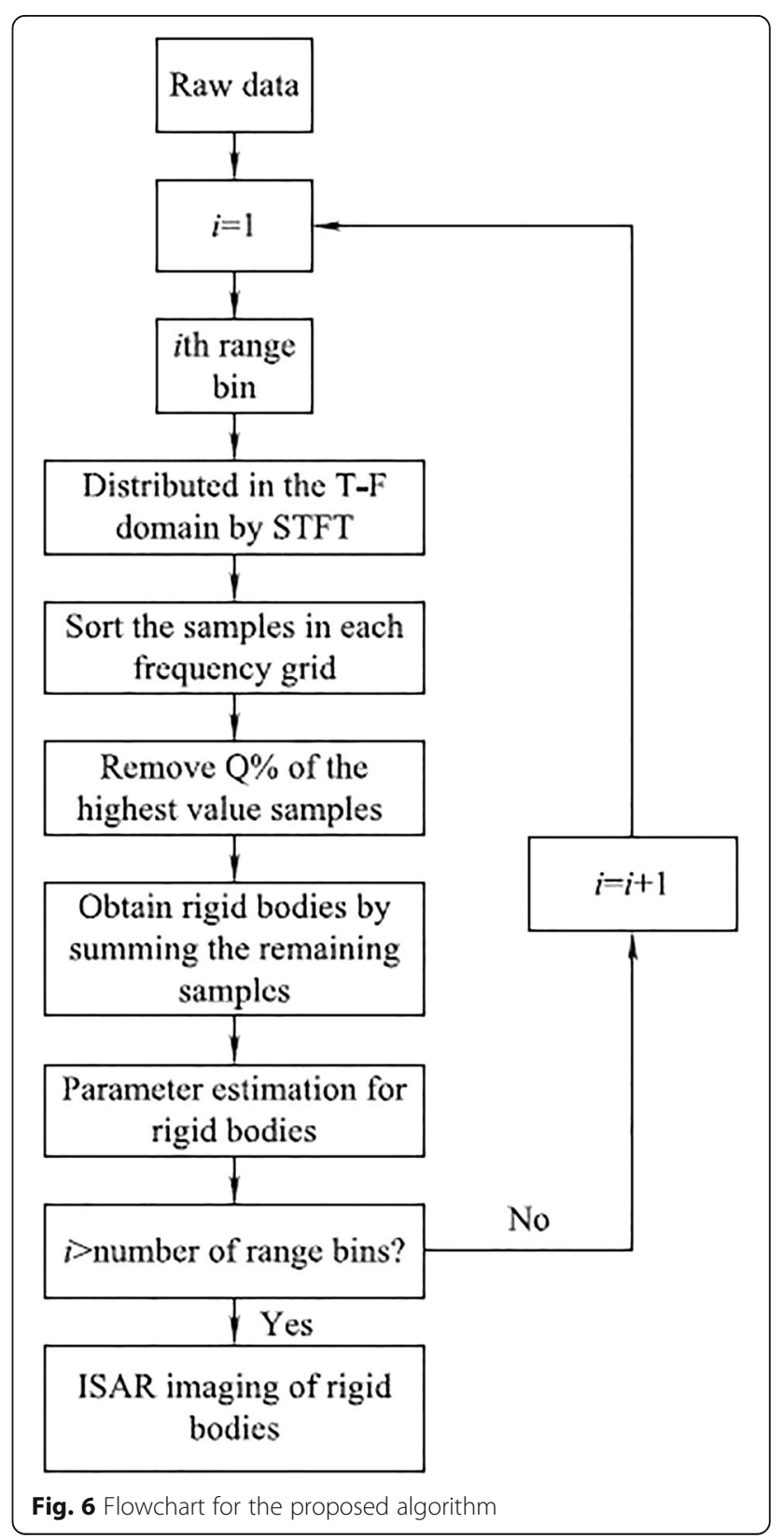



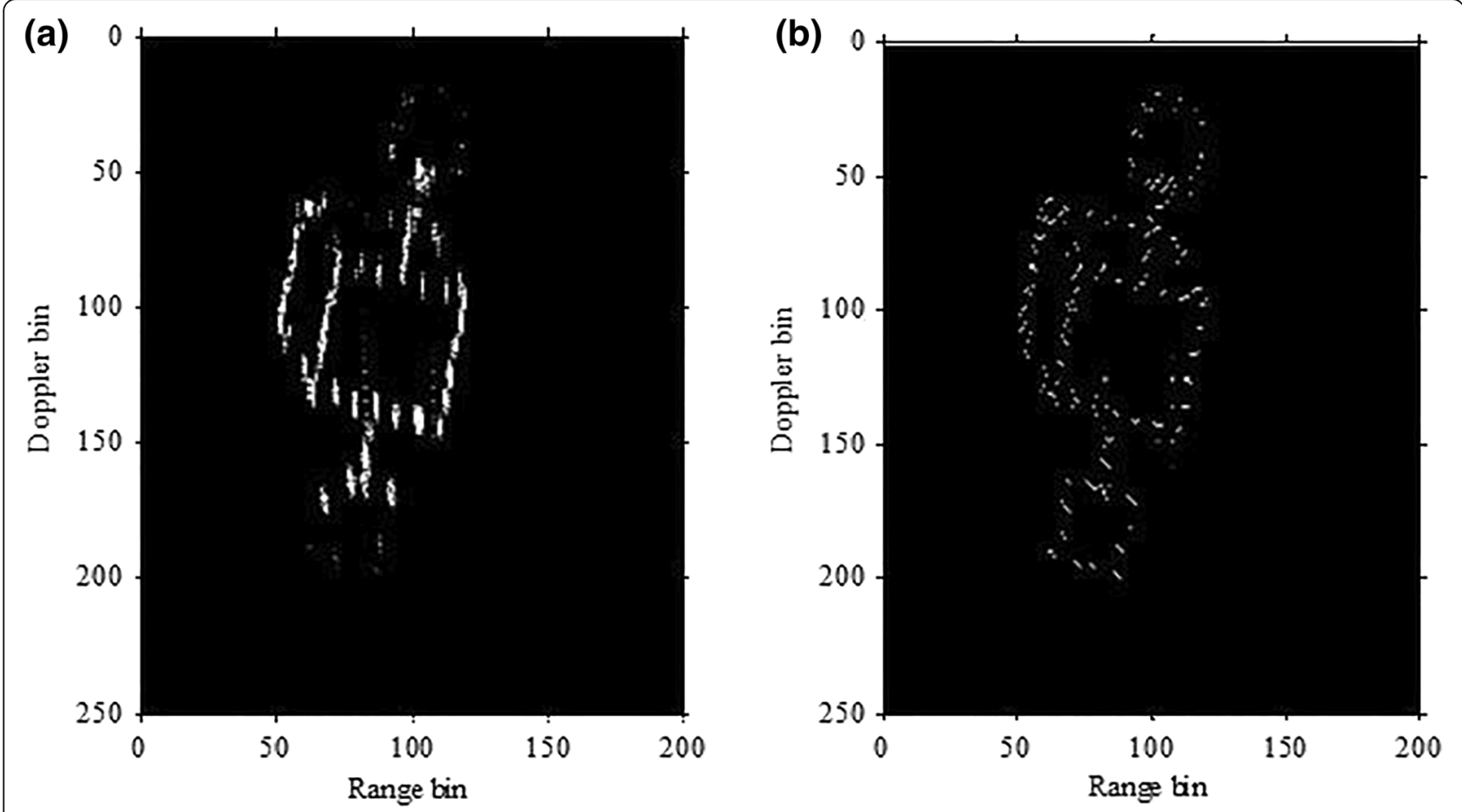

Fig. 7 Simulation results of the proposed algorithm. a Result of m-D removal algorithm in Section 4.1. $\mathbf{b}$ Result of the proposed algorithm

Step 5 For the $i$-th rigid body scattering point, the signal amplitude value is estimated by the expression $\sigma_{i}=S_{i L}(k) /$ $\left[W(0) \times M_{Q}\right]$ by finding the frequency value of the rigid body single-frequency signal estimated at the peak of $k_{i 0}$. In the time-frequency domain, the length of the rigid-body single-frequency signal should meet: $|l| \leq 1+2\left(M / M_{W}-1\right)$, where $M_{W}$ is the window length, $M / M_{W}-1$ is the number of STFT nonzero values of the single-frequency signal at $k$ $=k_{i 0}$. When $k=k_{i 0}$, find and note the position, set the value of STFT whose length is $M / M_{W}-1$ to zero on both sides. This process is repeated when $i=i+1$.

Step 6 Estimate all the frequency $\tilde{k}_{i 0}$ and amplitude values $\tilde{\sigma}_{i 0}$, obtain rigid body single-frequency signal for each distance unit, and then, use R-D imaging algorithm to obtain ISAR image of the target.

The flowchart for the proposed algorithm is shown in Fig. 6.

Using this algorithm, the low-orbit satellite of Section 4.1 is simulated and analyzed. The simulation parameters are the same as those in Section 4.1. The simulation results are shown in Fig. 6 . Through the comparison between Fig. $6 a, b$, by just the m-D removal method in Fig. 6a, smearing effects of rigid bodies caused by side lobes influence the imaging quality. It can be seen that rigid bodies in Fig. $6 \mathrm{~b}$ are clearly distinguished, which shows that the improved algorithm effectively solves the problem of rigid body information loss and obtains ISAR image of the satellite with good quality (Fig. 7).

\section{Conclusions}

When the space target has a micro-Doppler effect, the micro-motion will produce the frequency modulation of the radar echo, and its Doppler has the characteristics of sinusoidal frequency modulation. In this paper, the micro-spin model is modeled, and the echo is processed by short-time Fourier transform (STFT), Wigner-Vile Distribution (WVD), and pseudo-Wigner-Vile Distribution (PWV), and the micro-Doppler curve is derived. On this basis, for the space target with spin components, this paper uses the micro-Doppler removal ISAR imaging algorithm based on STFT to obtain good imaging results and proposes an improved algorithm based on rigid body parameter estimation which can obtain better ISAR image.

\section{Acknowledgements}

The authors would like to thank the anonymous reviewers for their valuable comments and suggestions to improve the quality of the article.

\section{Funding}

There are no sources of funding body reported for this manuscript.

Authors' contributions

All authors contributed to the work. All authors read and approved the final manuscript.

Competing interests

The authors declare that they have no competing interests. 


\section{Publisher's Note}

Springer Nature remains neutral with regard to jurisdictional claims in published maps and institutional affiliations.

Received: 5 July 2017 Accepted: 15 October 2017

Published online: 30 October 2017

\section{References}

1. VC Chen, L Fa yin, SH Shen, et al., Micro-Doppler effect radar: Phenomenon, model, and simulation study. IEEE Trans. Aerosp. Electron. Syst. 42(1), 2-21 (2006)

2. R Kleinman, R Mack, Scattering by linearly vibrating objects. IEEE Trans. Antennas Propag. 27(3), 344-352 (1979) Electronic, Systems, 2006, 42( 1): 2-21

3. T Thayaparan, S Abrol, E Riseborough, L Stanković, D Lamothe, G Duff, Analysis of radar micro-Doppler signatures from experimental helicopter and human data. IET Proc. Radar Sonar Navig 1(4), 288-299 (2007)

4. X Bai, F Zhou, M Xing, High resolution ISAR imaging of targets with rotating parts. IEEE Trans. Aerosp. Electron. Syst. 47(4), 2530-2543 (2011)

5. V. Popović, I. Djurović, L. Stanković, T. Thayaparan and M. Daković, Autofocusing of SAR images based on parameters estimated from the PHAF. Signal Processing. 90(5), 1382-1391 (2010)

6. H Gao, L Xie, S Wen, et al., Micro-Doppler signature extraction from ballistic target with micro-motions. IEEE Trans. Aerosp. Electron. Syst. 46(4), 19691982 (2010)

7. R Zhang, G Li, YD Zhang, Micro-doppler interference removal via histogram analysis in time-frequency domain. IEEE Trans. Aerosp. Electron. Syst. 52(2), 755-768 (2016)

8. M Ritchie, F Fioranelli, H Borrion, Multistatic micro-Doppler radar feature extraction for classification of unloaded/loaded micro-drones. IET Radar Sonar Navig 11(1), 116-124 (2017)

9. DP Fairchild, RM Narayanan, Multistatic micro-doppler radar for determining target orientation and activity classification. IEEE Trans. Aerosp. Electron. Syst. 52(1), 512-521 (2016)

\section{Submit your manuscript to a SpringerOpen ${ }^{\circ}$ journal and benefit from:}

- Convenient online submission

- Rigorous peer review

- Open access: articles freely available online

- High visibility within the field

- Retaining the copyright to your article 